\title{
The Notch target genes Hey1 and Hey2 are required for embryonic vascular development
}

\author{
Andreas Fischer, ${ }^{1}$ Nina Schumacher, ${ }^{1}$ Manfred Maier, ${ }^{1}$ Michael Sendtner, ${ }^{2}$ and Manfred Gessler ${ }^{1,3}$ \\ ${ }^{1}$ Theodor-Boveri-Institut fuer Biowissenschaften (Biozentrum), Physiologische Chemie I, Universitaet Wuerzburg, 97074 \\ Wuerzburg, Germany; ${ }^{2}$ Institut fuer Klinische Neurobiologie, Universitaet Wuerzburg, D-97072 Würzburg, Germany
}

The Delta-Notch signaling pathway plays a central role in the development of most vertebrate organs. The Hey family of bHLH transcription factors are direct targets of Notch signaling. Loss of Hey2 in the mouse leads to cardiac defects with high postnatal lethality. We have now generated a mouse Hey1 knockout that has no apparent phenotypic defect. The combined loss of Hey1 and Hey2, however, results in embryonic death after embryonic day 9.5 (E9.5) with a global lack of vascular remodeling and massive hemorrhage. Initial vasculogenesis appears unaffected, but all subsequently developing major vessels in the embryo and yolk sac are either small or absent. Furthermore, the placental labyrinth completely lacks embryonic blood vessels. Similar vascular defects are observed in Jagged1 and Notch1 knockout mice. In the latter we found Hey1 and Hey2 expression in yolk sacs to be strongly reduced. Remaining large arteries in both Notch1 and Hey1/Hey2 knockout mice fail to express the arterial endothelial markers CD44, neuropilin1, and ephrin-B2. This indicates that $\mathrm{Hey} 1 / \mathrm{Hey} 2$ are essential transducers of Notch signals in cardiovascular development that may mediate arterial cell fate decision.

[Keywords: Notch pathway; angiogenesis; vasculogenesis; arteriogenesis; Hey1; Hey2]

Supplemental material is available at http://www.genesdev.org.

Received November 16, 2003; revised version accepted March 18, 2004.

The vascular system is the first organ to function in the developing vertebrate embryo. There are two fundamentally different processes that establish blood vessels: initial vasculogenesis generates a primitive network of vessels through aggregation and tube formation of angioblast precursor cells. Angiogenesis then leads to further growth, branching, and remodeling of the vascular tree (Risau 1997). These processes are followed by additional maturation steps to recruit smooth muscle and mural cells. They are controlled by several signaling molecules, most notably the vascular endothelial growth factor (VEGF) and angiopoietin system, but also more common factors such as PDGF-B and TGF- $\beta$ (Carmeliet 2003). Most of these factors are used in setting up as well as in subsequent maintenance and adaptation of a regular vascular bed.

There is a clear distinction between the arterial and venous sides of the circulatory system. Although not immediately evident by morphology in the early embryo, there are striking differences in gene expression patterns between arterial and venous endothelia even before the

${ }^{3}$ Corresponding author.

E-MAIL gessler@biozentrum.uni-wuerzburg.de; FAX 49-931-888-4150. Article and publication are at http://www.genesdev.org/cgi/doi/10.1101/ gad.291004. onset of circulation, suggesting that there must be separate genetic programs. The prime example is the ephrinB2 (arterial) and EphB4 (venous) ligand and receptor pair (Wang et al. 1998). More recently, the neuropilin $1 / 2$ coreceptors, or members of the Notch ligand and receptor family, were also shown to exhibit vessel-specific expression (for review, see Adams 2003). Knockout studies have shown that these genetic determinants of arterial or venous identity are essential for proper formation of the vasculature in the developing embryo.

The zebrafish gridlock mutation has provided an additional candidate for specifying the arterial lineage, with the venous one representing the default pathway (Zhong et al. 2000, 2001). Mutant fish display impaired maturation of the aorta due to a hypomorphic mutation of the gridlock (grl) gene, a hairy-related basic helix-loop-helix transcription factor that represents the zebrafish ortholog of mammalian Hey2. In morpholino knockdown studies of the $g r l$ gene, the maturation defect can lead to a complete loss of arterial marker expression and even a lack of the aorta. This led to the postulate that grl is necessary to specify arterial fates in undetermined vascular progenitor cells.

Hairy and Enhancer-of-split-related basic helix-loophelix (bHLH) transcription factors such as grl and Her in zebrafish, or Hey and Hes in mammals, represent the 
main transducers of Notch signals during development (Iso et al. 2003). They can act as transcriptional repressors, and they have been implicated in a large number of developmental processes from Drosophila up to higher vertebrates. Especially in somitogenesis and neurogenesis, a prominent role for Her and Hes genes could be demonstrated. The role of Hey genes remained less clear up to now, however. All three Hey genes (Hey1, Hey2, and $H e y L)$ are expressed in dynamic patterns in multiple tissues of the mouse embryo (Leimeister et al. 1999, 2000b; Nakagawa et al. 1999|. The cycling expression in the presomitic mesoderm of Hey2 initially suggested an involvement in the somitogenesis clockwork (Leimeister et al. 2000a). The knockout of Hey2, also known as Hesr2/CHF1/Hrt2/Herp1, revealed a critical function during heart development with ventricle septum defect, persistent foramen ovale, tricuspid valve stenosis, and cardiomyopathy as the predominant anomalies (Donovan et al. 2002; Gessler et al. 2002; Sakata et al. 2002). Most of the affected mice died during the first week of life. For the other two Hey genes, no knockout phenotype has been reported thus far.

We have generated a Hey1 knockout allele in the mouse to study the role of Hey1. Here we show that loss of Hey1 does not result in a discernible phenotypic defect. The combined loss of Hey1 and Hey2, however, leads to a lethal vascular defect that affects the placenta, the yolk sac, and the embryo itself, likely due to impaired arterial fate determination and maturation. Our data identify both $\mathrm{Hey}$ genes as essential effectors within the Notch signaling cascade in mammalian vascular development.

\section{Results}

Generation of $\mathrm{Heyl}^{-/-}$mice

The murine Hey1 locus comprises five exons and spans a genomic distance of $6 \mathrm{kbp}$ (Steidl et al. 2000). A $\lambda$ phage clone (SV3) encompassing the entire locus was used to construct a lacZ replacement vector for gene targeting in R1 embryonic stem (ES) cells (Fig. 1A). The lacZ-neo cassette was fused in frame to exon 1 with a subsequent deletion of Hey1 exons 2-4 and part of exon 5. After electroporation, drug selection, and screening by PCR and Southern blot, a single clone could be identified that had undergone successful gene targeting. This clone (LZ1F1) was used to generate chimeric mice that transmitted the deleted Hey1 allele through the germline. Heterozygous offspring expressed $\beta$-galactosidase activity in a pattern that is very similar to that obtained by mRNA in situ hybridization in control mice (data not shown). These mice did not show any obvious phenotypic anomaly up to now, on the initial mixed 129SVJ/ C57BL/6 background, or after further backcross to $\mathrm{C} 57 \mathrm{BL} / 6$.

When heterozygous mice were interbred, wild-type, heterozygous, and homozygous offspring were born at expected Mendelian ratios. We have not identified any major developmental defect in these Hey1 knockout (KO) mice as yet. Specifically, all organ systems with prominent expression of Hey1 during development, such as somites, kidney, heart atria, and nervous tissue (Leimeister et al. 1999) do not show evidence of developmental delay or subsequent impaired function. This suggests that complete loss of Hey1 function is well tolerated
Figure 1. Knockout of the Hey1 locus and embryonic death of Hey1/2 DKO embryos. (A) The targeting construct is depicted above the mouse genomic Hey 1 locus containing five exons. Homology regions of $3 \mathrm{kbp}$ (BglII/Ecl136II) and $1.4 \mathrm{kbp}$ (HindIII) containing mainly promoter and 3'untranslated regions, respectively, were used for recombination. The PGK-neo cassette (Neo) is flanked by loxP sites (triangles) for subsequent removal by cre-recombinase. The HSV-TK (TK) cassette was included for negative selection. Flanking genomic probes used for Southern blot hybridization are highlighted by thick lines. (B) BglII; (E) Ecl136II; (H) HindIII; (*) stop codon; (pBS) pBluescript cloning vector backbone. (B) At E10.5, the Hey1/2 DKO embryos can be clearly identified. They are much smaller compared to a het/het littermate (fourth embryo), and they lack prominent mes- and telencephalic vesicles $\left({ }^{*}\right)$. The extensive pericardial sac is highlighted by arrows. $(C, D) \mathrm{Al}$ ready at E9.5, most DKO embryos suffer from massive hemorrhage in the trunk (arrowheads, $C$ ) or the pericardial sac $(D)$.

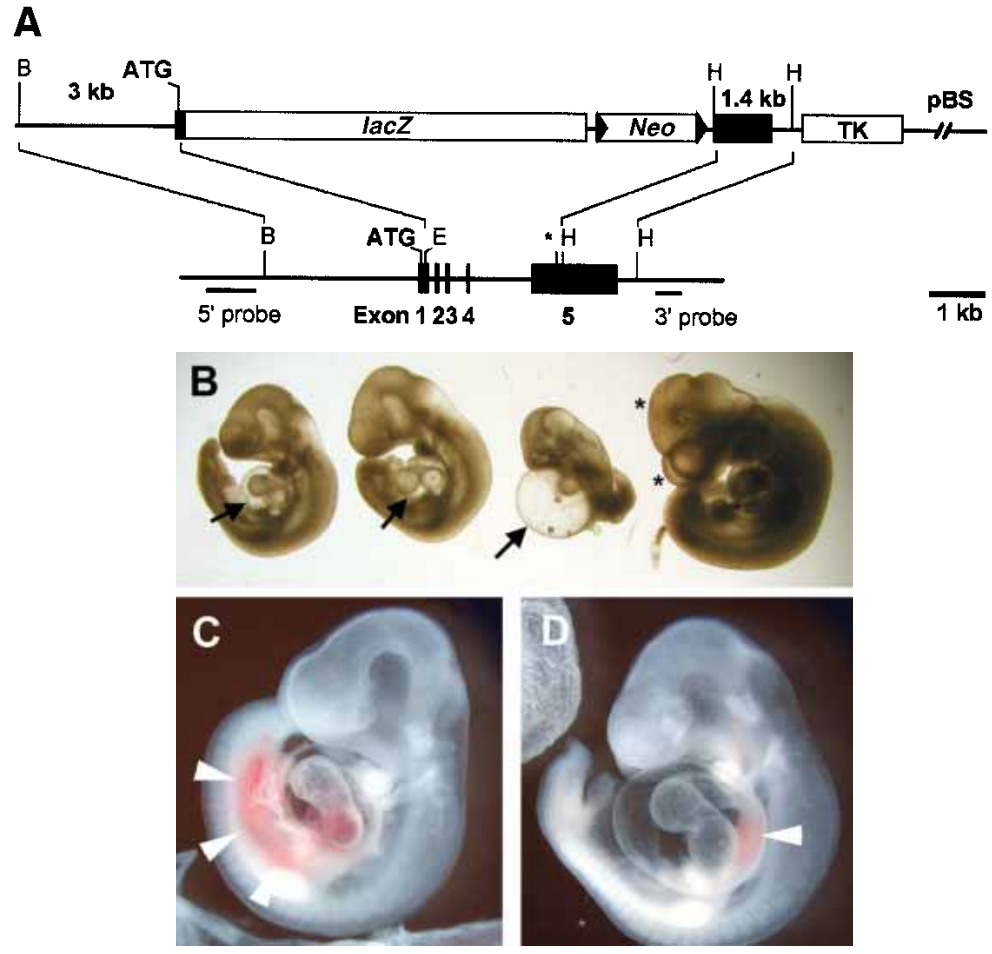


under standard breeding conditions. It is possible that this lack of phenotypic alteration is partly due to redundancy in the expression of Hey gene family members, although there is only limited overlap with the patterns detected for Hey2 and HeyL (Leimeister et al. 1999, 2000b).

\section{Mice with combined Hey1/2 loss are not viable}

Deletion of the Hey2 gene in mice leads to high postnatal lethality due to ventricle septum defects and frequent additional cardiac anomalies (Donovan et al. 2002; Gessler et al. 2002; Sakata et al. 2002). Whole-mount in situ hybridization with Heyl and HeyL probes did not reveal expanded expression domains for these genes in Hey2 $^{-/-}$embryos at different stages (data not shown). Nevertheless, such hybridizations still represent a rather coarse level of analysis and cannot rule out redundancy. Therefore, we started to intercross Hey1 and Hey2 KO mice to clarify potential additive effects of a combined loss of both genes.

Double heterozygous mice were fertile and phenotypically normal. Breeding of these mice to generate doubleknockout (DKO) mice revealed a striking deviation from expected inheritance, as shown in Table 1 . Hey $1^{+-}$/ $\mathrm{Hey}^{-/-}$mice were severely underrepresented and also showed additional unexplained adult lethality. This may in part be due to an additive effect of the loss of a single Hey1 allele on top of a Hey2 KO genotype. A more striking difference was seen in the case of Hey1 KO mice, a genotype that shows no functional impairment, where the additional loss of a single Hey2 allele reduced survival by $\sim 60 \%$. The reason for this increased embryonic lethality is unclear and must be investigated in more detail in the future. Nevertheless, a small fraction of these $\mathrm{Hey}^{-/-} / \mathrm{Hey}^{2^{+/}}$mice survived and could be used for subsequent breeding. The complete loss of both Hey1 and Hey2 is not compatible with life, as we did not detect any surviving Hey1/Hey2 double-knockout (Hey1/2 DKO) offspring, either at birth or at weaning.

Analysis of consecutively earlier embryonic stages revealed a fraction of resorbed Hey1/2 DKO concepti at embryonic day 14.5 (E14.5). Hey1/2 DKO embryos that approximately resembled their littermates with respect to size and developmental stage could be found only at E9.5. Nevertheless, most of them already showed severe defects (Fig. 1B-D). Thereafter, development of these embryos did not progress, and they increasingly deterio- rated. At E10.5 all Hey1/2 DKO embryos were strongly affected. They exhibited significantly reduced overall size, and all were characterized by balloon-like pericardial sacs and pale yolk sacs, suggestive of cardiovascular failure. Most of these embryos likely die due to massive hemorrhage in the head, the trunk, and the pericardial cavity. More detailed analysis revealed that multiple organ systems are affected in these embryos, as presented below. At E11.5-E12.5, only dead or massively retarded embryos were found, with most of them already in the process of resorption (data not shown).

\section{Heyl/2 function is necessary for placental labyrinth development}

The placenta is essential for gas and nutrient exchange between embryo and mother during most of gestation. Placental development begins after the fusion of the chorionic plate and allantois around E8.5. Buds of allantoic cells including blood vessel precursors invade the chorionic plate, and branching morphogenesis is initiated. Together with the expansion of the trophoblast compartment, the labyrinthine space is established. Both Hey genes are highly expressed in the allantois, which likely reflects the high abundance of endothelial precursors in this structure (Leimeister et al. 1999). Chorioallantoic fusion takes place in Hey1/2 DKO embryos, and formation of an umbilical cord begins. This initial circulation soon ceases, however, and after E9.5 the connection between embryo and placenta does not mature into a thicker umbilical cord. The placenta is still of expected size at E10.5, but histological analysis revealed severe structural changes (Fig. 2). The entire labyrinthine layer appears devoid of fetal blood circulation. Marker gene expression was analyzed to characterize the defect in more detail. The outermost trophoblast layer, the giant cells, express Csh1 (placental lactogen 1), and the spongiotrophoblast cells are positive for Tpbpa (also known as 4311) as expected (Fig. $2 \mathrm{C}-\mathrm{F}$ ). The labyrinthine layer, however, appears extremely cell-rich, in contrast to the extensive intermingling of maternal and embryonic blood spaces in controls (Fig. 2G-I). Although maternal erythrocytes are still present in blood sinus, there is no expression of Vegfr2, a marker that identifies the endothelia-containing embryonic blood vessels. Vegfr2 staining was similar in Hey $1 / 2$ DKO and control placentas only in the allantois and chorionic plate. Closer examination of the chorionic plate revealed that the initial

Table 1. Frequencies of genotypes obtained from Hey1/Hey2 matings

\begin{tabular}{|c|c|c|c|c|c|c|c|c|c|}
\hline Age and parental genotypes & $\mathrm{KO} / \mathrm{KO}$ & $\mathrm{KO} /$ het & $\mathrm{KO} / \mathrm{wt}$ & het/KO & het/het & het/wt & $\mathrm{wt} / \mathrm{KO}$ & wt/het & $w t / w t$ \\
\hline $\mathrm{P} 21-\mathrm{P} 28$ het $/$ het $\times$ het $/$ het & $0^{\mathrm{a}}(5)$ & $3(10)$ & $9(5)$ & $2(10)$ & $28(20)$ & $16(10)$ & $3(5)$ & $10(10)$ & $4(5)$ \\
\hline P21-P28 KO/het $\times$ het $/$ het & $0^{\mathrm{a}}(6)$ & $6(12)$ & $10(6)$ & $2(6)$ & $22(12)$ & $8(6)$ & - & - & - \\
\hline E9.5-E11.5 KO/het $\times$ het $/$ het & $12^{\mathrm{b}}(11)$ & $17(22)$ & $13(11)$ & $9(11)$ & $25(22)$ & $10(11)$ & - & - & - \\
\hline
\end{tabular}

Expected numbers (rounded) are given in parentheses.

Age is given as days postnatal (P21-P28) or postconception (E9.5-E11.5).

${ }^{\text {a}}$ Never observed in any other subsequent litter.

${ }^{b}$ Nine of twelve embryos were severely retarded or dead and showed internal bleeding and aberrant yolk sac vasculature. 


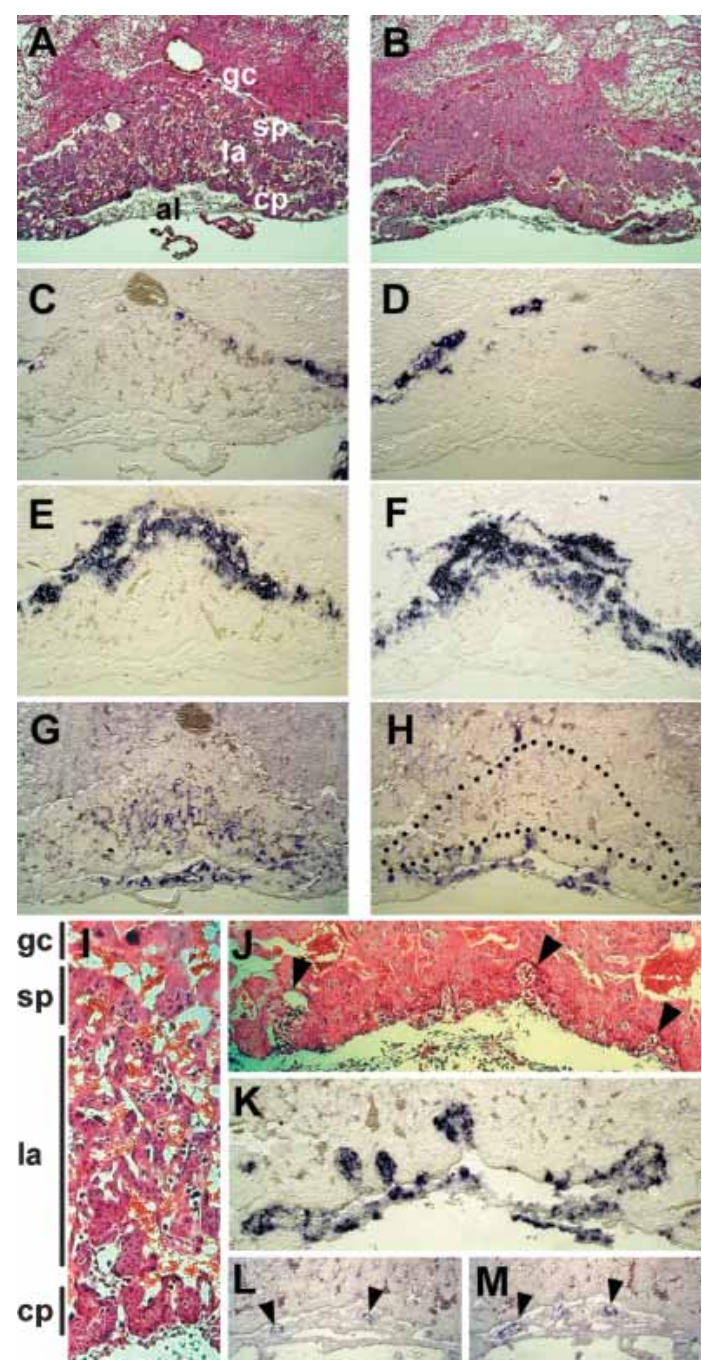

Figure 2. Placental defects in Hey1/2 DKO mice. A layered structure is visible at E10.5 in both normal $(A, C, E, G, I)$ and $H e y 1 / 2$ DKO $(B, D, F, H)$ placentas. $(A, B) \mathrm{H} \& \mathrm{E}$ staining shows a close intermingling between maternal blood spaces with small erythrocytes and embryonic vessels with larger, nucleated red blood cells in the control placenta. Especially the labyrinth in the DKO placenta appears cell-rich and devoid of embryonic vessels. $(C, D)$ The giant cell border between the maternal and the embryonic compartment stains positive with a Csh1 (placental lactogen 1) probe. (E,F) Tpbpa (4311) marks the spongiotrophoblast layer that appears unaltered in DKO placentas. $(G, H)$ Vegfr2 (Flk1) stains endothelia of all embryonic vessels in the labyrinth and the chorioallantoic plate. A dotted line highlights the labyrinthine region in DKO mutants, which lacks any staining. $(J, K)$ Fetal vessels only start to invade the trophoblast layer, but fail to branch and extend any further as seen by H\&E $(J)$ and endothelial Vegfr2 (K) staining. Abortive buds are marked by triangles. (I) In a control placenta, the labyrinthine layer is characterized by intermingling and close apposition of maternal and embryonic blood spaces to facilitate nutrient and gas exchange. $(L, M)$ Both Hey1 and Hey2 are expressed in endothelia of embryonic vessels in the chorioallantoic plate (arrowheads) of controls and faintly if at all in the embryonic labyrinth. (gc) Giant cells; (sp) spongiotrophoblast; (la) labyrinth; (cp) chorionic plate; (al) allantois. buds are formed, and these do contain Vegfr2-positive blood vessels, but further invasion of the labyrinth does not occur (Fig. 2J,K). Interestingly, Hey1 and Hey2 are both expressed in blood vessels of the allantois and chorionic plate in normal placentas (Fig. 2L,M). The limited sensitivity of in situ hybridization does not permit a definitive answer as to whether there is any expression in endothelia of the fine labyrinthine network in controls. Nevertheless, the normal thickness of the trophoblast compartment in Hey1/2 DKO placentas would argue against an inherent trophoblast proliferative defect, and rather suggests that vascularization from the embryonic side does not occur.

\section{Remodeling defects in Hey1/2 DKO yolk sacs}

The yolk sac is the site of primary hematopoiesis and also the first site of blood vessel development from clusters of blood islands. The blood vessels form de novo through aggregation of endothelial precursor cells in a process called vasculogenesis to form a primitive network, the primary capillary plexus. Thereafter, remodeling and pruning set in to generate an ordered and interconnected tree of arterial and venous vessels.

In Hey1/2 DKO embryos, the yolk sacs show severe changes that are already visible at E9.5 in most cases and can be used in a fairly reliable fashion to identify DKO embryos (Fig. 3). The initial vascular plexus is present in all DKO embryos at E9.5, with blood-filled vascular structures, which provides evidence of efficient erythropoiesis. In controls a similar network can be seen, but there, a branched structure of larger vessels can already be detected. One day later, a highly organized vascular bed has been established with mature vitelline arteries and veins. In most of the E10.5 Hey1/2 DKO yolk sacs, however, the primitive vascular plexus is either unchanged or has even started to degenerate. Histological sections revealed a fairly regular initial structure of the yolk sac, and PECAM staining of endothelia confirmed the presence of functional vascular structure, albeit with poor or absent remodeling in mutants. There is a lack or at least a rapid degeneration of vitelline vessels in all mutants. At E11.5, when most embryos are already necrotic, the endodermal and mesodermal layers separate, and no vascular structures are left in the yolk sac.

Despite this rapid degeneration of Hey1/2 DKO yolk sacs, blood flow must have been established between the yolk sac vasculature and the embryo proper, as all Hey1/2 DKO embryos have circulating red blood cells that are easily visible during preparation and in histological sections. Because embryonic erythropoiesis only sets in after E11, these erythrocytes must have originated from the yolk sac as the primary site of hematopoiesis.

\section{Vascular defects in Hey1/2 DKO embryos}

Inspection of Hey1/2 DKO embryos already showed evidence of hemorrhage in E9.5 and especially in E10.5 em- 

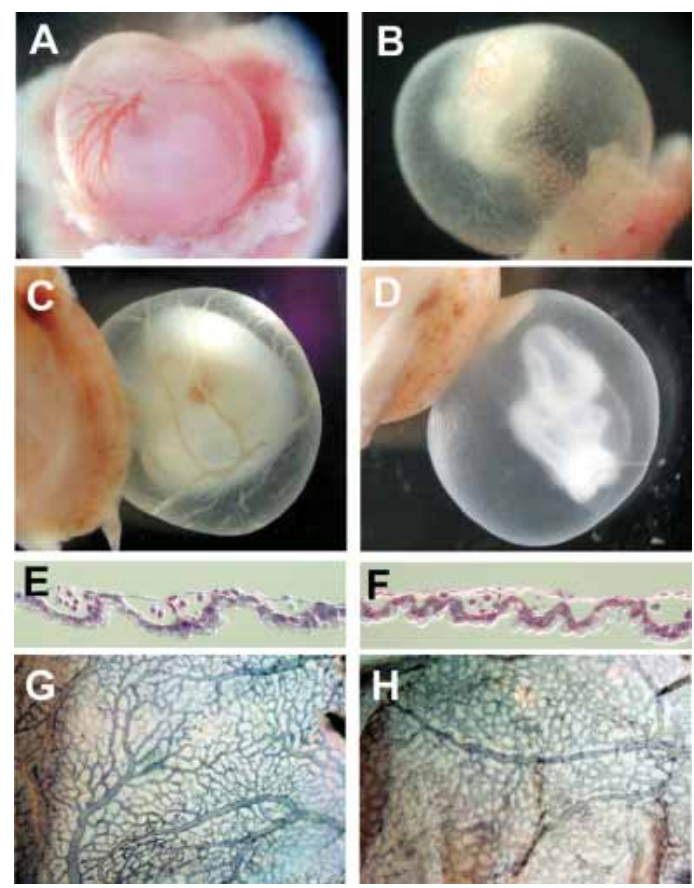

Figure 3. Yolk sac defects in Hey1/2 DKO mice. There is a clear difference between intact het/het controls $(A, C)$ and DKO $(B, D)$ yolk sacs. $(A, B)$ At E9.5, a vascular network with embryonic red blood cells can be seen. In $A$, large vessels are clearly visible, whereas DKO embryos only exhibit the primitive vascular network without further reorganization. $(C, D)$ At E10.5, large vitelline vessels are present in controls, but in DKO embryos the yolk sacs are pale and begin to degenerate. $(E, F)$ Sections of E9.5 yolk sacs clearly show that initial vasculogenesis is comparable in het/het control $(E)$ and DKO $(F)$ embryos. $(G, H)$ The presence of blood cells in sections and intact yolk sacs suggests that hematopoiesis from blood islands is not affected. PECAM staining of blood vessel endothelia confirms highly organized vascular branches in controls $(G)$, but complete absence of remodeling in yolk sacs from a DKO littermate $(H)$.

bryos. Therefore, the vascular system is likely affected in the embryo proper as well. Whole-mount staining with PECAM antibodies revealed successful vasculogenesis throughout the embryo with a dense network of superficial and deep blood vessels (Fig. 4A-D). However, in Hey1/2 DKO embryos the vascular pattern appears rather coarse, indicating that angiogenetic remodeling was impaired. Especially in the head region, vessels are truncated and do not form a finely branched tree. The intersomitic vessels that form through angiogenetic sprouting do appear, but again the pattern of vessels is less organized and ordered compared to control embryos.

Transverse sections were used to visualize major blood vessels in E9.5 embryos (Fig. 4E,F). Generally, DKO embryos appeared developmentally retarded. The neural tube was thinner, and surrounding mesenchymal cells appeared sparse. Although the heart initiated looping in most embryos, the myocardium was much thinner and lacked a trabecular region in all sections. It is currently unclear whether this represents a specific cardiac defect or rather reflects the global developmental delay.
Most striking, however, was the partial lack of large blood vessels. Although variable among DKO embryos, the paired dorsal aortae and cardinal veins were frequently either missing or strongly reduced on one or even both sides. This was not due to a lack of endothelial precursors, as we could detect numerous cells positive for Vegfr1/2 (Flt1 and Flk1) or the vascular endothelial marker VE-Cadherin (Fig. 4G,H) that formed either smaller vessels or were part of the reduced major vessels. There were few if any vascular sprouts in the neural tube, but again, this may be due to the general developmental delay.

Nevertheless, the aortic vessels begin proper maturation, as they are ensheathed by cells positive for SM22, a marker for smooth muscle cells (Fig. 4I-L). Wholemount in situ hybridization analysis showed that the aorta contains smooth muscle cells along its entire length. However, SM22 staining around these aortae appears weaker and in some cases does not appear to form a complete circle, suggesting that this process of aortic wall formation is impaired and may account for subsequent leakage.

The global impairment of blood supply and circulation is expected to lead to embryonic hypoxia, and in many sections staining for Vegf mRNA appeared stronger in DKO embryos (data not shown). As such analyses are difficult to quantitate by in situ hybridization, we employed real-time RT-PCR analysis. Comparison of E9.5 embryos (five DKO and four controls) revealed a 2.3-fold increase in Vegf mRNA ( $p=0.001)$. There was no change in the amounts of Notch1, Dll1, Dll4, VE-Cadherin, Vezf1, Ang1, Eklf, or Neuropilin1/2 transcripts. Thus, a lack of Hey1/2 leads to the induction of an embryonic Vegf response, likely due to global hypoxia.

\section{Vascular defects are not due to a lack of circulation}

The highly dynamic process of angiogenesis in the developing embryo depends on a functional blood circulation, as unused vessel branches may rapidly be purged. Thus, the lack of a heart beat may result in subsequent deterioration of blood vessels. Hey1 and Hey2 are both expressed in nonoverlapping domains in early cardiac precursors, with Hey1 restricted to atrial and Hey2 to ventricular cardiomyocytes. Hey2 functional defects only manifest after E13.5, with ventricular septum defect and other morphogenetic problems, whereas deletion of Hey1 apparently does not visibly disturb heart development. In Hey1/2 DKO embryos, the hearts are formed and they begin to loop. The myocardium remains very thin, but this may reflect the general growth retardation in these embryos. Full enlargement of the ventricular portion does not take place, but blood flow could be detected in most E9.5 and some E10.5 DKO embryos during preparation. To assess cardiac function in a more quantitative manner, we explanted hearts at E9.5 and cultured them for several days. Heart rate was measured daily. There was no significant variation in beat frequency between hearts with different Hey1/Hey2 genotypes laverage beats per minute \pm standard deviation; 
Fischer et al.

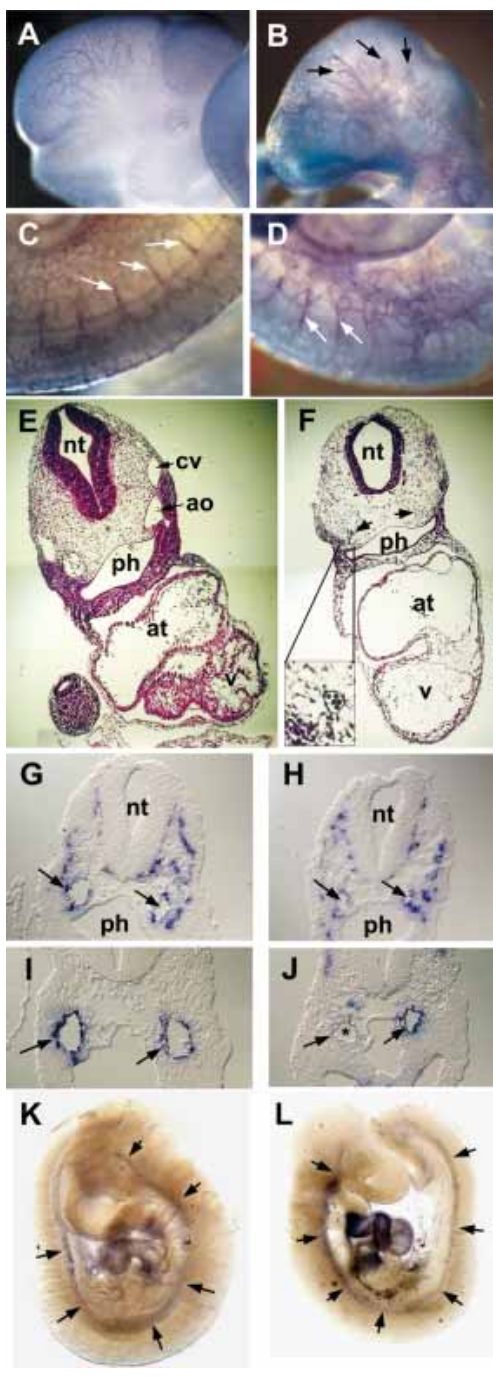

Figure 4. Vascular defects in Hey1/2 DKO embryos. $(A-D)$ Comparison of whole-mount PECAM antibody staining of E10.5 control $(A, C)$ and DKO $(B, D)$ embryos reveals intact vasculogenesis. Large cranial vessels appear truncated in mutants (arrows). Angiogenetic sprouts of intersomitic vessels (white arrows) are present, but the vascular pattern in the trunk is rather coarse. $(E, F) \mathrm{H} \& \mathrm{E}$-stained cross-sections revealed reduction or loss of aorta (arrows) and cardinal vein. The myocardial wall is thinner in mutants, and ventricular trabeculation is missing. The neural tube is thinner and the mesenchymal compartment is cell-poor in mutants. $(G, H)$ In situ hybridization with the endothelial marker VE-Cadherin identifies the aorta (arrows) and multiple smaller vessels. $(I, J)$ The smooth muscle cell marker SM22 stains the aorta in controls $(I)$, but in DKO embryos $(J)$ staining is variable with partial or even complete loss $\left({ }^{*}\right)$ of the hybridization signal. $(K, L)$ Whole-mount in situ hybridization for SM22 shows that the aorta is associated with smooth muscle cells along its length in controls and mutants, at least at this level of resolution. (ao) Aorta; (at) atrium; (cv) cardinal vein; (nt) neural tube; (ph) pharynx; (v) ventricle.

wild type: $126 \pm 26$; het/het: $128 \pm 18$; het/KO: $133 \pm 14$; KO/het: $127 \pm 19$; KO/KO: $141 \pm 17 ; n=3-12$ each). Thus, a basal level of blood circulation appears to be possible, suggesting that vascular degeneration does not result from cardiac insufficiency.

\section{A similar vascular phenotype of Heyl/2 DKO and Notch1 KO embryos}

In many respects, the Hey1/2 DKO embryos resemble KO embryos for Jag1 or Notch1 (Xue et al. 1999; Krebs et al. 2000). All of these mutants show similar defects in yolk sac vascular remodeling, massive embryonic hemorrhages, enlarged pericardial sacs, absence or loss of large embryonic blood vessels, and impaired placental development. Death occurs at almost the same time point in all three cases. Jag1, Notch1, Hey1, and Hey2 are all coexpressed in the arterial endothelium, suggesting that this is the primary affected cell type (Villa et al. 2001; Fischer and Gessler 2003). On a molecular level we found a sixfold increase in Vegf mRNA expression $(p=0.02)$ in Notch1 KO embryos at E9.5, similar to DKO embryos (see above) that is most likely due to hypoxia.

To further support a link between Notch signaling and Hey gene expression in vascular development, we analyzed yolk sacs of Notch1 KO mice by real-time RTPCR. Blood vessels and precursors make up a large fraction of cells there, and yolk sacs can almost be used as a surrogate for blood vessels. Both, Hey1 and Hey2 are expressed in control yolk sacs as expected, but in those obtained from Notch1 KO mice, transcript levels were strongly reduced by a factor of 17.9 and 8.3, respectively $(p<0.01)$. This supports the notion that Hey1 and Hey2 together mediate Notch1 effects on yolk sac vascular remodeling.

The Notch pathway genes D111, D113, and Notch1 are required for somitogenesis and subsequent establishment of somite polarity. We showed previously that Hey2 exhibits cycling expression in the presomitic mesoderm and may thus be part of the somitogenesis clockwork, together with other bHLH factors of the Hes/Her family (Leimeister et al. 2000a). Hey1 expression also shows dynamic variation, but limited to the anterior presomitic mesoderm. In maturing somites, all three Hey genes are expressed in the caudal half of each somite, but presomitic and somitic expression of Hey genes is largely lost in Notch1 and Dll1 KO embryos. Therefore, it appeared likely that somitogenesis may be perturbed in Hey1/2 DKO embryos. Although somite formation in Hey1/2 DKO embryos ceases around E9.5 after 21-26 somites, there were no morphological alterations such as fused or aberrantly sized somites. Expression of the marker gene uncx4.1 that identifies the caudal half of all somites was unaltered in comparison to control embryos (see Supplementary Fig. 1). This would argue against a critical role of Hey1/2 function in the presomitic mesoderm. Nevertheless, in newly formed somites, remaining HeyL function may still conceal a potential requirement for Hey gene function.

It was recently reported that Notch1 and Dll1 mutant mice show laterality defects with randomization of heart looping (Krebs et al. 2003; Przemeck et al. 2003). Although Hey1/2 DKO hearts appeared underdeveloped, 
we did not find any evidence of left-sided looping. Thus, Hey1/2 do not appear to be involved in mediating laterality effects of Dll1 and Notch1.

\section{Hey genes and arterial fate}

A knockdown of grl (Hey2) activity in zebrafish leads to an aortic maturation defect and a loss of arterial marker expression (Zhong et al. 2001). Further, it was shown that in zebrafish Notch signaling is essential for arterial versus venous cell fate decisions. The role of gridlock in this process remains controversial, however (for review, see Torres-Vazquez et al. 2003). Here we examined expression of ephrin-B2, CD44 (Wheatley et al. 1993), and neuropilin1, which are all established arterial endothelial markers, to assess vascular lineage identity in our embryos. Antibody staining revealed strong expression of these proteins in the aorta, but not in the cardinal vein of wild-type and Hey1/2 double heterozygous embryos at stages E9.5 and E10.5. In contrast, no or only strongly reduced staining could be seen in the aorta of Hey1/2 DKO embryos (Fig. 5). This suggests that in mice, combined Hey1 and Hey2 function is essential for establishing arterial cell fate or identity.

To further define the role of Notch signaling in arte-

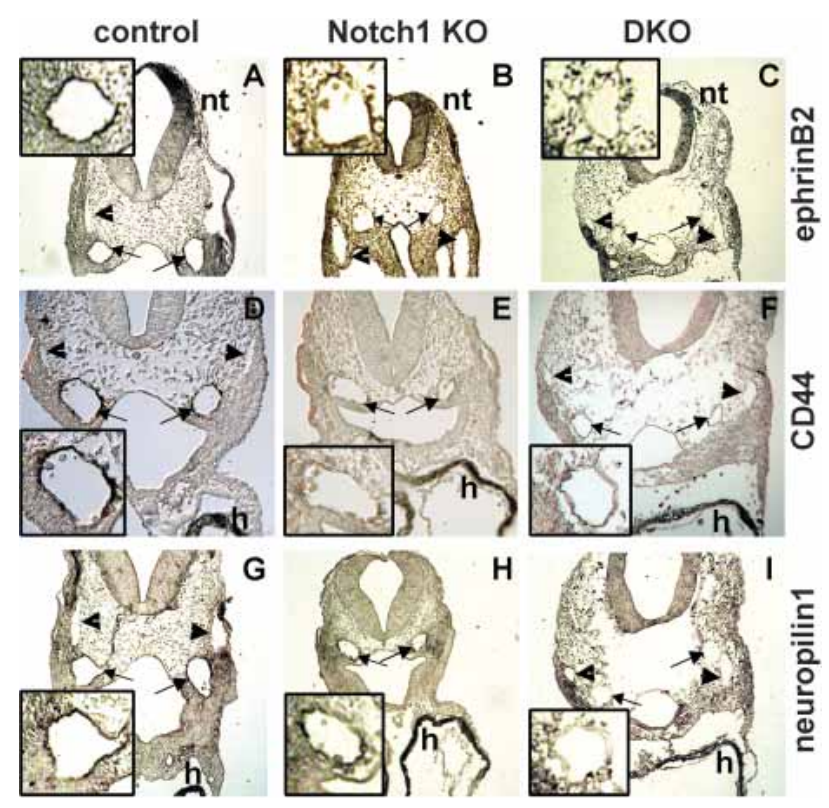

Figure 5. Lack of vascular ephrin-B2, CD44, and neuropilin1 expression in Notch1 and Hey1/2 KO embryos. Expression of the arterial endothelial markers ephrin-B2 $(A-C)$, CD44 $(D-F)$, and neuropilin1 $(G-I)$ in wild-type $(A, D, G)$, Notch1 $1^{-/-}(B, E, H)$, and Hey1/2 DKO $(C, F, I)$ embryos at E9.5. Immunohistochemistry of transverse sections reveals staining of both aortae (arrows) in control embryos, whereas the cardinal veins (arrowheads) are negative. Similar sections of the mutant embryos still exhibit cardiac CD44 and neuropilin1 expression as well as ephrin-B2 staining in the neural tube, but the aortae are clearly not stained. (h) Heart; (nt) neural tube. Enlarged views of an aortic vessel for each case are shown in insets. rial/venous development, we re-examined Notch1 KO embryos. Again, we found no or strongly reduced arterial expression of CD44, neuropilin1, and ephrin-B2 in Notch1 KO embryos (Fig. 5). Loss of these markers in Notch1 KO and Hey1/2 DKO embryos seems to occur specifically in the aorta, as we detected normal CD44 and neuropilin 1 expression in the myocardium and ephrin-B2 in the neural tube.

To extend these findings of common vascular phenotypic effects, we analyzed E9.5 yolk sacs of Notch1 KO mice (seven KO, 22 control littermates) and Hey1/2 DKO mice (five DKO, 19 control littermates) with quantitative RT-PCR. We found a significant down-regulation of the arterial specific marker ephrin-B2 in the yolk sacs of Notch1 and Hey1/2 DKO deficient mice (4.2- and 6.1-fold, $p<0,01)$. The transcript level of its receptor EphB4, which is expressed more highly in veins, was not altered in both cases. Taken together, these results strongly suggest that in mice the correct development and differentiation of arterial endothelial cells from vascular precursors depends on functional Notch1 and Hey1/2 signaling.

\section{Discussion}

The specific and dynamic expression pattern of Hey1 in the developing mouse embryo suggested several sites where Hey1 may play an essential role (Leimeister et al. 1999). Examples are the expression of Hey1 during somite, branchial arch, and kidney development. The mutually exclusive expression of Hey 1 and Hey2 in the atria and ventricles of the heart is also quite intriguing, because Hey2 loss leads to ventricular septum and valve defects (Fischer et al. 2002). Nevertheless, deletion of Hey1 in the mouse does not result in any major developmental or functional impairment. Although subtle defects may still have gone unnoticed, the normal lifespan, fertility, and behavior with regular morphological and histological appearance of many organs argue against a major functional defect. Furthermore, the partly overlapping expression of other Hey family members or perhaps even partial functional redundancy with Hes family genes may contribute to mask critical tasks.

Proof of partial redundancy of Hey gene function is now provided with our analysis of Hey1/Hey2 DKO embryos, which die after E9.5 with global vascular deficiencies that are not seen in KOs of either Hey1 or Hey2 alone. There are several mouse KO lines that exhibit lethal vascular defects between E8.5 and E12.5 (for review, see Thurston 2003). Examples are KO mice for members of the VEGF pathway (Vegf, Vegfr1-3), Ang1/ Tie2, ephrin-B2/EphB4, Notch pathway genes (Notch1, Jag1, Pofut, PS1/2), the MAP kinase and Smad pathways (e.g., ERK5, Smad5, Alk1, Endoglin), and other less well categorized genes such as CD148 or WAVE2.

Interestingly, the spectrum of tissues affected can be variable. It is rarely described that blood vessels in all compartments, that is, the yolk sac, the placental labyrinth, and the embryo proper are defective. This may indicate that the defect in our Hey1/2 DKO mice must 
reside primarily within the blood vessels, that is, endothelia, or less likely in smooth muscle cells, but not in surrounding tissue-specific supporting cells. This is nicely exemplified in the placenta, where we found a largely normal morphological structure, presence of all histological compartments, and basically unaltered gene expression patterns. All trophoblastic cells seem to differentiate correctly and even maternal blood sinus is present, but there is a lack of embryo-derived blood vessels. This is in contrast to many other $\mathrm{KO}$ situations with placental defects, where there is a clear size reduction of different layers and a compromised function of trophoblast cells (for review, see Rossant and Cross 2001).

\section{The Notch pathway as a vascular signaling module}

The importance of Notch signaling for vascular development has now been established at every level of the cascade, based on knockout approaches in the mouse. Although there are often additional nonvascular anomalies, which may contribute to the observed lethality, these will not be discussed here. As depicted schematically in Figure 6, there is a proven requirement in vascular development for the Jag1 ligand, which is expressed in endothelia and smooth muscle cells (Xue et al. 1999). It has been proposed that Dll4 should be the preferred Notch ligand at the earliest embryonic stages, but this has not been verified by $\mathrm{KO}$ analysis as yet (Krebs et al. 2000; Shutter et al. 2000). On the receptor side, Notch1 appears to be most important. The somewhat stronger phenotype of Notch1/4 double mutants suggests that at least to some extent parallel pathways do exist, but Notch4 deficiency on its own does not impair vascular development (Krebs et al. 2000). The presence of such vascular defects in Pofut and PS1/PS2 KO mice further suggests that correct glycosylation and cleavage of Notch receptors is likewise essential (Donoviel et al. 1999; Shi and Stanley 2003).

There is only a small number of target genes to mediate Notch function, and most of them belong to the Hes and Hey gene families (Iso et al. 2003). Published KO phenotypes for several Hes genes do not include vascular defects, but rather present with somitogenesis and neurogenesis problems (Kageyama and Ohtsuka 1999). Our finding that a combined loss of Hey1 and Hey2 phenocopies the vascular defects seen in Jag1 and Notch1 KO mice suggests that this pair of Hey genes represents a critical and essential transducer of Notch signals in vascular development. When we tested Notch1 KO yolk sacs by real-time RT-PCR analysis, we found a strongly reduced expression of Hey1 and Hey2, which supports our interpretation of $\mathrm{Hey} 1 / 2$ being the primary effectors of Notch signals in vascular development.

The early lethality of our Hey1/2 DKO mice precludes analysis of later functions during maintenance and remodeling of the vascular system during adult life. The presence of Notch3 mutations in patients with CADASIL (cerebral autosomal dominant arteriopathy with subcortical infarcts and leukoencephalopathy) suggests that appropriate Notch function is still needed in later life in humans (Joutel et al. 1996). This has been further substantiated through the analysis of a transgenic mouse model, where a typical CADASIL point mutation was introduced into the Notch3 locus (Ruchoux et al. 2003), resulting in a CADASIL-like phenotype. In this context it may be instructive to conditionally inactivate one or both Hey genes in adult mice and assess adult vascular functions.

\section{Hey1/2 loss and the gridlock phenotype}

The divergent phenotypes of the zebrafish gridlock mutant and the mouse Hey2 knockout was difficult to explain initially. Whereas gridlock leads to an aortic maturation defect that has been proposed to resemble aortic coarctation, the mouse Hey2 knockout affects primarily the heart with ventricular and atrial septal defects, valve anomalies, and cardiomyopathy (Zhong et al. 2000; Gessler et al. 2002). A morpholino knockdown of grl in zebrafish had shown that grl is essential to confer an arterial phenotype on vascular precursor cells; otherwise arterial marker expression would be suppressed and the vessels disintegrated, implicating $g r l$ in arterial fate specification (Zhong et al. 2001). It should be noted, however, that this model has been questioned by others (Lawson et al. 2002; Torres-Vazquez et al. 2003). Those authors found direct evidence of a Shh-VEGF-Notch signaling cascade in zebrafish that mediates arterial fate decision. Importantly, a lack of Notch activity led to reduced arterial marker expression with still unaltered grl levels, suggesting that grl acts in aortic development, but perhaps not in primary fate decision.

We were able to show that expression domains of Hey genes are quite different in zebrafish and mice (Winkler

Figure 6. Notch signal transduction in endothelial biology. KO studies in the mouse identified a complete scenario for Notch signal transduction in arterial endothelial cells. The Jag1 (and presumably also the Dll4) ligand appear critical for endothelial cells and their precursors. Notch1, aided by Notch4, transmits the signal, dependent on it own glycosylation and cleavage by Pofut and Ps $1 / 2$. The Notch intracellular domain together with RBP-Jk activates Hey 1 and Hey2 transcription. Knockout of any of these components leads to very similar vascular deficiencies.

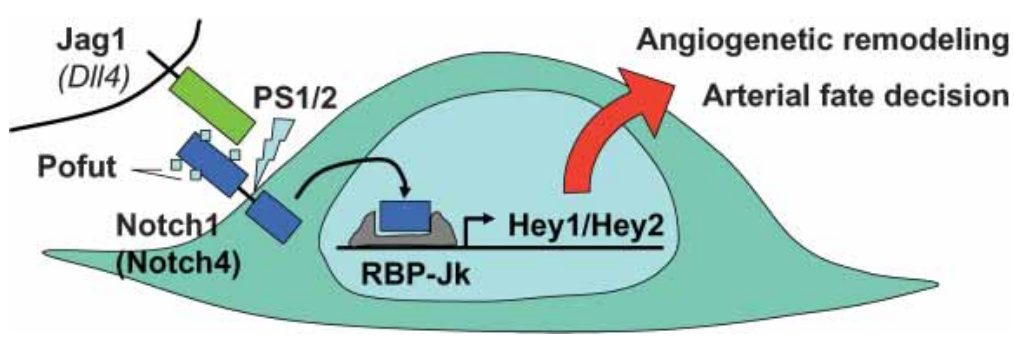


et al. 2003). Whereas grl is the only Hey gene family member expressed in blood vessels in fish, all three Hey genes are expressed in the vascular compartment in the mouse, opening up the possibility for complementation (Fischer et al. 2002). Indeed, the phenotype of Hey1/2 DKO mice described here is rather similar to the grl knockdown, thus restoring a consistent picture of Hey gene function being essential for vascular morphogenesis. This further suggests that the third Hey gene, HeyL, is not able to compensate for Hey1/2 loss in blood vessels, despite its prominent expression (Leimeister et al. 2000b). An important difference between mouse and zebrafish may lie in the fact that loss of Notch1 reduces Hey1 and Hey2 levels in endothelial-rich yolk sacs, whereas Notch blockade in fish did not change arterial grl levels.

\section{Hey gene function and arterial fate}

Although it is tempting to propose that Hey1 and Hey2 together specify arterial fate in angioblast precursors, this is not yet formally proven, and it has not been ruled out that Hey genes function subsequently in arterial endothelial maturation. Interestingly, we found a loss of expression of the arterial endothelial markers CD44, neuropilin1, and ephrin-B2 in our Hey1/Hey2 KO mice. This was seen even in those E9.5 DKO embryos that still presented fairly normal-sized aortas, where tissues had not yet undergone massive deterioration. This clearly points to a lack of arterial characteristics in the aorta.

During embryonic development there is a clear preference for Hey1 and Hey2 expression in arteries as opposed to veins (Fischer et al. 2002). This is in line with the arterial-predominant expression of Notch pathway genes (Villa et al. 2001) and the fact that Hey gene expression can be induced by Notch signals (Maier and Gessler 2000; Iso et al. 2002). A recent microarray comparison of a large series of human endothelial cell lines confirmed an arterial-specific expression for Hey2 (Chi et al. 2003). Further, those authors showed that ectopic expression of Hey2 in HUVEC cells specifically induces expression of a series of genes that were characteristic for arterial endothelia in their analyses, implicating Hey2 as a key regulator of the arterial phenotype.

Based on in vitro culture models, Hey1 has been proposed as a modulator of the endothelial phenotype, especially the switch between proliferation and differentiation (Henderson et al. 2001; Taylor et al. 2002). This has been postulated to involve repression of Vegfr2 expression. Although it is too early to draw a conclusive picture of the role of Hey 1 from these data, it is clear that in vivo at least one of these two Hey genes is needed for correct vascular development. As neither Hey1 nor Hey2 KO mice exhibit overt vascular deficiencies, these genes seem to act redundantly in vascular development.

The finding that Hey genes can also be induced by the BMP-Smad pathway (Korchynskyi et al. 2003) introduces additional layers of complexity. It is now well established that the Alk1-Smad5 pathway is critical for angiogenesis (for review, see Waite and Eng 2003) and thus, Hey gene activity may not only be dependent on Notch signals, but could also integrate information from additional sources. This is further supported by the recent report on synergistic induction of Heyl by Notch and BMP, where Heyl inhibits endothelial cell migration (Itoh et al. 2004).

Our identification of Hey1 and Hey2 as essential mediators of Notch functions in blood vessel morphogenesis clearly extends the current concept of arteriogenesis. The challenge for future experiments will be to identify vascular Hey gene targets that mediate the effects needed to generate robust and functional arteries. Furthermore, it will be instructive to test animal models with defects in the Alk1-Smad pathway for aberrant Hey gene regulation that would position $\mathrm{Hey}$ genes as common integrators for angiogenetic signals.

\section{Materials and methods}

\section{Generation of Heyl knockout mice}

The gene structure of Hey1, based on $\lambda$ clone SV3, has been described (Steidl et al. 2000). A Hey1-lacZ targeting vector was constructed to delete the coding region of mouse Hey1 including exons 2-4 and parts of exons 1 and 5. The lac $Z$ gene was fused in frame with part of exon 1 encoding the first 10 amino acids. The vector pKSTK-loxPneo provided floxed PGK-neo and HSV-TK selection markers.

R1 ES cells were electroporated with linearized vector, and after drug selection 300 clones were picked. These were screened by PCR and Southern blotting with flanking and internal hybridization probes. Clone LZ1F1 showed homologous recombination of a single-copy integrate and was used to derive chimeric mice after injection into C57BL/6 blastocysts. All subsequent breeding was done with $\mathrm{C} 57 \mathrm{BL} / 6$ mice to generate congenic mice. The floxed PGK-neo cassette was removed by breeding once with a CMV-cre transgenic line. Subsequent generations were selected to be free of the cre transgene.

\section{Mouse breeding and genotyping}

The Hey2 and Notch1 KO strains have been described (Radtke et al. 1999; Gessler et al. 2002). Both Hey1 and Hey2 KO lines were interbred only after at least five generations of backcross with C57BL/6 mice. Mouse tail tips or portions of yolk sacs or embryos were used for genotyping by PCR. Tissues were digested in $200 \mu \mathrm{L} 100 \mathrm{mM} \mathrm{NaCl}, 0.5 \%$ sodium lauroylsarcosine, $50 \mu \mathrm{g} / \mathrm{mL}$ Proteinase $\mathrm{K}$, and $5 \%$ Chelex-100 overnight at $55^{\circ} \mathrm{C}$. Lysates were boiled for $8 \mathrm{~min}$, and $1 \mu \mathrm{L}$ aliquots were used for genotyping. Hey1 status was tested with primers HeyllacKOtest5' (CGTCCGCCACCATGAAGA), clk2 (CTGGCCAAA ACCTGGGAC), and Z3L (ATCGGTGCGGGCCTCTTCGCT ATTA). Products are 300 bp (wild type) and 240 bp (lacZ knockin allele). Primer combinations for Hey2 have been described (Gessler et al. 2002).

\section{$R T-P C R$}

Total RNA was extracted from complete yolk sacs using Trizol reagent (Invitrogen) according to the manufacturer's instructions. Aliquots of $2 \mu \mathrm{g}$ RNA were used for reverse transcription (First-strand kit, Fermentas) with oligo-dT priming. Real-time PCR reactions with SybrGreen quantification were set up with 
$1 / 25$ of each cDNA preparation in a Bio-Rad iCycler. Relative expression levels and statistical significance were calculated based on an Hprt standard using REST software (Pfaffl et al. 2002). Essentially similar results were obtained by normalizing to Tbp expression levels. All amplicons (80-180 bp) spanned at least one intron and showed efficient amplification that allowed us to equate one threshold cycle difference as representing a twofold expression difference. Primer pairs were Hprt (TGT TGTTGGATATGCCCTTG, ACTGGCAACATCAACAGGA CT), Tbp (GTGGATAGGGAAGGCAGGA, TCAAACCCAG AATTGTTCTCC), Vegf (GGCTTTACTGCTGTACCTCCA, ACAGGACGGCTTGAAGATGTA), Hey1 (TGAGCTGAGAA GGCTGGTAC, ACCCCAAACTCCGATAGTCC), Hey2 (TG AGAAGACTAGTGCCAACAGC, TGGGCATCAAAGTAGC CTTTA), ephrin-B2 (GCGGGATCCAGGAGATCCCCACTTG GACT, GTGCGCAACCTTCTCCTAAG), and EphB4 (GCGG GATTCCAGCGCTCTGGACAAGATGAT, CATCTCAAAG GAGCCGAATC). Primer combinations used for other, noninformative genes are available upon request.

\section{Histological analysis}

For routine histological analysis, tissues were fixed in $4 \%$ paraformaldehyde overnight and embedded in paraffin wax for sectioning. Generally, 4- $\mu \mathrm{m}$ sections were cut and stained with Cole's haemalaun and $0.5 \%$ Hemalum and Phloxine.

\section{In situ hybridization and immunochemistry}

Details of RNA in situ hybridizations on whole mount or sectioned embryos were described (Leimeister et al. 1998). Diglabeled riboprobes were generated from vectors provided by others, or pCS2P clones containing amplified cDNA segments as indicated: Hey1, Hey2 (Leimeister et al. 1999), Vegfr1/2 (pFlt-1, pflk-1, G. Breier), uncx4.1 (pSVSport1-Uncx4.1, A. Kispert), SM22 (nt 488-995 of NM_011526), Tpbpa (4311; nt 52-555 of NM_009411), Csh1 (Placental lactogen 1; nt 122-722 of NM_ 008864), VE-Cadherin (Cdh5; nt 708-1312 of NM_009868).

Endothelial cell staining of whole-mount preparations was performed using PECAM antibody MEC13.3 (a gift from G. Breier) as described in Vecchi et al. (1994). CD44 staining on paraffin sections with antibody IM7 (J. Sleeman, Karlsruhe) and neuropilin1 staining with Protein A purified serum (provided by A. Kolodkin, Baltimore, MD) was done as described in Wheatley et al. (1993) using the Vectastain Elite ABC kit (Vector Laboratories). Ephrin-B2 antibody (R\&D Systems) staining was done according to Batlle et al. (2002).

\section{Embryo heart culture}

Hearts from E9.5 embryos were cultured essentially as described by Noveroske et al. (2002). Briefly, hearts were dissected and grown on 1\% agarose in DMEM medium containing 10\% fetal calf serum. Heart rate was counted daily for $5 \mathrm{~d}$.

\section{Acknowledgments}

We thank C. Winkler, M. Schartl, G. Breier, L. Hein, C. Englert, and all lab members for stimulating discussions; F. Radtke (Epalinges) for generously providing the Notch1 KO line; J. Sleeman for the CD44 (IM7); and A. Kolodkin for the neuropilin 1 antiserum. We thank B. Klamt, A. Winkler, and A. Klaus for expert technical assistance and mouse colony maintenance, and B. Holtmann for KO support. This work was supported by
SFB465 (TP A4) and a grant from the Deutsche Forschungsgemeinschaft (Ge539/9-1).

The publication costs of this article were defrayed in part by payment of page charges. This article must therefore be hereby marked "advertisement" in accordance with 18 USC section 1734 solely to indicate this fact.

\section{References}

Adams, R.H. 2003. Molecular control of arterial-venous blood vessel identity. J. Anat. 202: 105-112.

Batlle, E., Henderson, J.T., Beghtel, H., van den Born, M.M., Sancho, E., Huls, G., Meeldijk, J., Robertson, J., van de Wetering, M., Pawson, T., et al. 2002. Beta-catenin and TCF mediate cell positioning in the intestinal epithelium by controlling the expression of EphB/ephrinB. Cell 111: 251-263.

Carmeliet, P. 2003. Angiogenesis in health and disease. Nat. Med. 9: 653-660.

Chi, J.T., Chang, H.Y., Haraldsen, G., Jahnsen, F.L., Troyanskaya, O.G., Chang, D.S., Wang, Z., Rockson, S.G., van de Rijn, M., Botstein, D., et al. 2003. Endothelial cell diversity revealed by global expression profiling. Proc. Natl. Acad. Sci. 100: 10623-10628.

Donovan, J., Kordylewska, A., Jan, Y.N., and Utset, M.F. 2002. Tetralogy of fallot and other congenital heart defects in Hey2 mutant mice. Curr. Biol. 12: 1605-1610.

Donoviel, D.B., Hadjantonakis, A.K., Ikeda, M., Zheng, H., Hyslop, P.S., and Bernstein, A. 1999. Mice lacking both presenilin genes exhibit early embryonic patterning defects. Genes \& Dev. 13: 2801-2810.

Fischer, A. and Gessler, M. 2003. Hey genes in cardiovascular development. Trends Cardiovasc. Med. 13: 221-226.

Fischer, A., Leimeister, C., Winkler, C., Schumacher, N., Klamt, B., Elmasri, A., Steidl, C., Maier, M., Knobeloch, K.P., Amann, K., et al. 2002. Hey bHLH factors in cardiovascular development. Cold Spring Harb. Symp. Quant. Biol. 67: 6370.

Gessler, M., Knobeloch, K.P., Helisch, A., Amann, K., Schumacher, N., Rohde, E., Fischer, A., and Leimeister, C. 2002. Mouse gridlock: No aortic coarctation or deficiency, but fatal cardiac defects in Hey2 $2^{-/}$mice. Curr. Biol. 12: 16011604.

Henderson, A.M., Wang, S.J., Taylor, A.C., Aitkenhead, M., and Hughes, C.C. 2001. The basic helix-loop-helix transcription factor HESR1 regulates endothelial cell tube formation. $J$. Biol. Chem. 276: 6169-6176.

Iso, T., Chung, G., Hamamori, Y., and Kedes, L. 2002. HERP1 is a cell type-specific primary target of Notch. J. Biol. Chem. 277: 6598-6607.

Iso, T., Kedes, L., and Hamamori, Y. 2003. HES and HERP families: Multiple effectors of the Notch signaling pathway. $J$. Cell Physiol. 194: 237-255.

Itoh, F., Itoh, S., Goumans, M.J., Valdimarsdottir, G., Iso, T., Dotto, G.P., Hamamori, Y., Kedes, L., Kato, M., and Dijke Pt, P. 2004. Synergy and antagonism between Notch and BMP receptor signaling pathways in endothelial cells. EMBO $J$. 23: 541-551.

Joutel, A., Corpechot, C., Ducros, A., Vahedi, K., Chabriat, H., Mouton, P., Alamowitch, S., Domenga, V., Cecillion, M., Marechal, E., et al. 1996. Notch3 mutations in CADASIL, a hereditary adult-onset condition causing stroke and dementia. Nature 383: 707-710.

Kageyama, R. and Ohtsuka, T. 1999. The Notch-Hes pathway in mammalian neural development. Cell Res. 9: 179-188. 
Korchynskyi, O., Dechering, K.J., Sijbers, A.M., Olijve, W., and ten Dijke, P. 2003. Gene array analysis of bone morphogenetic protein type I receptor-induced osteoblast differentiation. J. Bone Mine.r Res. 18: 1177-1185.

Krebs, L.T., Xue, Y., Norton, C.R., Shutter, J.R., Maguire, M., Sundberg, J.P., Gallahan, D., Closson, V., Kitajewski, J., Callahan, R., et al. 2000. Notch signaling is essential for vascular morphogenesis in mice. Genes \& Dev. 14: 1343-1352.

Krebs, L.T., Iwai, N., Nonaka, S., Welsh, I.C., Lan, Y., Jiang, R., Saijoh, Y., O'Brien, T.P., Hamada, H., and Gridley, T. 2003. Notch signaling regulates left-right asymmetry determination by inducing Nodal expression. Genes \& Dev. 17: $1207-$ 1212.

Lawson, N.D., Vogel, A.M., and Weinstein, B.M. 2002. Sonic hedgehog and vascular endothelial growth factor act upstream of the Notch pathway during arterial endothelial differentiation. Dev. Cell 3: 127-136.

Leimeister, C., Bach, A., and Gessler, M. 1998. Developmental expression patterns of mouse sFRP genes encoding members of the secreted frizzled related protein family. Mech. Dev. 75: 29-42.

Leimeister, C., Externbrink, A., Klamt, B., and Gessler, M. 1999. Hey genes: A novel subfamily of hairy- and Enhancer of splitrelated genes specifically expressed during mouse embryogenesis. Mech. Dev. 85: 173-177.

Leimeister, C., Dale, K., Fischer, A., Klamt, B., Hrabe de Angelis, M., Radtke, F., McGrew, M.J., Pourquie, O., and Gessler, M. 2000a. Oscillating expression of c-Hey2 in the presomitic mesoderm suggests that the segmentation clock may use combinatorial signaling through multiple interacting bHLH factors. Dev. Biol. 227: 91-103.

Leimeister, C., Schumacher, N., Steidl, C., and Gessler, M. 2000b. Analysis of HeyL expression in wild-type and Notch pathway mutant mouse embryos. Mech. Dev. 98: 175-178.

Maier, M.M. and Gessler, M. 2000. Comparative analysis of the human and mouse Heyl promoter: Hey genes are new Notch target genes. Biochem. Biophys. Res. Commun. 275: 652660.

Nakagawa, O., Nakagawa, M., Richardson, J.A., Olson, E.N., and Srivastava, D. 1999. HRT1, HRT2, and HRT3: A new subclass of bHLH transcription factors marking specific cardiac, somitic, and pharyngeal arch segments. Dev. Biol. 216: $72-84$.

Noveroske, J.K., Lai, L., Gaussin, V., Northrop, J.L., Nakamura, H., Hirschi, K.K., and Justice, M.J. 2002. Quaking is essential for blood vessel development. Genesis 32: 218-230.

Pfaffl, M.W., Horgan, G.W., and Dempfle, L. 2002. Relative expression software tool (REST) for group-wise comparison and statistical analysis of relative expression results in real-time PCR. Nucleic Acids Res. 30: e36.

Przemeck, G.K., Heinzmann, U., Beckers, J., and Hrabe de Angelis, M. 2003. Node and midline defects are associated with left-right development in Delta1 mutant embryos. Development 130: 3-13.

Radtke, F., Wilson, A., Stark, G., Bauer, M., van Meerwijk, J., MacDonald, H.R., and Aguet, M. 1999. Deficient T cell fate specification in mice with an induced inactivation of Notch1. Immunity 10: 547-558.

Risau, W. 1997. Mechanisms of angiogenesis. Nature 386: 671674.

Rossant, J. and Cross, J.C. 2001. Placental development: Lessons from mouse mutants. Nat. Rev. Genet. 2: 538-548.

Ruchoux, M.M., Domenga, V., Brulin, P., Maciazek, J., Limol, S., Tournier-Lasserve, E., and Joutel, A. 2003. Transgenic mice expressing mutant Notch3 develop vascular alterations characteristic of cerebral autosomal dominant arteriopathy with subcortical infarcts and leukoencephalopathy. Am. J. Pathol. 162: 329-342.

Sakata, Y., Kamei, C.N., Nakagami, H., Bronson, R., Liao, J.K., and Chin, M.T. 2002. Ventricular septal defect and cardiomyopathy in mice lacking the transcription factor CHF1/ Hey2. Proc. Nat1. Acad. Sci. 99: 16197-16202.

Shi, S. and Stanley, P. 2003. Protein O-fucosyltransferase 1 is an essential component of Notch signaling pathways. Proc. Nat1. Acad. Sci. 100: 5234-5239.

Shutter, J.R., Scully, S., Fan, W., Richards, W.G., Kitajewski, J., Deblandre, G.A., Kintner, C.R., and Stark, K.L. 2000. Dll4, a novel Notch ligand expressed in arterial endothelium. Genes \& Dev. 14: 1313-1318.

Steidl, C., Leimeister, C., Klamt, B., Maier, M., Nanda, I., Dixon, M., Clarke, R., Schmid, M., and Gessler, M. 2000. Characterization of the human and mouse HEY1, HEY2, and HEYL genes: Cloning, mapping, and mutation screening of a new bHLH gene family. Genomics 66: 195-203.

Taylor, K.L., Henderson, A.M., and Hughes, C.C. 2002. Notch activation during endothelial cell network formation in vitro targets the basic HLH transcription factor HESR-1 and downregulates VEGFR-2/KDR expression. Microvasc. Res. 64: $372-383$.

Thurston, G. 2003. Role of Angiopoietins and Tie receptor tyrosine kinases in angiogenesis and lymphangiogenesis. Cell Tissue Res. 314: 61-68.

Torres-Vazquez, J., Kamei, M., and Weinstein, B.M. 2003. Molecular distinction between arteries and veins. Cell Tissue Res. 314: 43-59.

Vecchi, A., Garlanda, C., Lampugnani, M.G., Resnati, M., Matteucci, C., Stoppacciaro, A., Schnurch, H., Risau, W., Ruco, L., Mantovani, A., et al. 1994. Monoclonal antibodies specific for endothelial cells of mouse blood vessels. Their application in the identification of adult and embryonic endothelium. Eur. J. Cell Biol. 63: 247-254.

Villa, N., Walker, L., Lindsell, C.E., Gasson, J., Iruela-Arispe, M.L., and Weinmaster, G. 2001. Vascular expression of Notch pathway receptors and ligands is restricted to arterial vessels. Mech. Dev. 108: 161-164.

Waite, K.A. and Eng, C. 2003. From developmental disorder to heritable cancer: It's all in the BMP/TGF- $\beta$ family. Nat. Rev. Genet. 4: 763-773.

Wang, H.U., Chen, Z.F., and Anderson, D.J. 1998. Molecular distinction and angiogenic interaction between embryonic arteries and veins revealed by ephrin-B2 and its receptor EphB4. Cell 93: 741-753.

Wheatley, S.C., Isacke, C.M., and Crossley, P.H. 1993. Restricted expression of the hyaluronan receptor, CD44, during postimplantation mouse embryogenesis suggests key roles in tissue formation and patterning. Development 119: 295306.

Winkler, C., Elmasri, H., Klamt, B., Volff, J.N., and Gessler, M. 2003. Characterization of hey bHLH genes in teleost fish. Dev. Genes Evol. 213: 541-553.

Xue, Y., Gao, X., Lindsell, C.E., Norton, C.R., Chang, B., Hicks, C., Gendron-Maguire, M., Rand, E.B., Weinmaster, G., and Gridley, T. 1999. Embryonic lethality and vascular defects in mice lacking the Notch ligand Jagged1. Hum. Mol. Genet. 8: 723-730.

Zhong, T.P., Rosenberg, M., Mohideen, M.A., Weinstein, B., and Fishman, M.C. 2000. Gridlock, an HLH gene required for assembly of the aorta in zebrafish. Science 287: 18201824.

Zhong, T.P., Childs, S., Leu, J.P., and Fishman, M.C. 2001. Gridlock signalling pathway fashions the first embryonic artery. Nature 414: 216-220. 


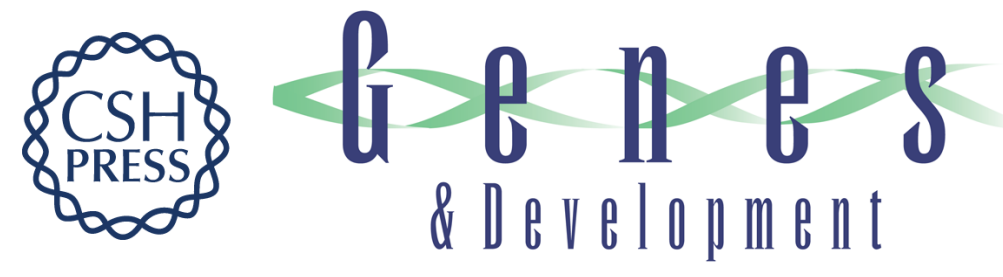

\section{The Notch target genes Hey1 and Hey2 are required for embryonic vascular development}

Andreas Fischer, Nina Schumacher, Manfred Maier, et al.

Genes Dev. 2004, 18:

Access the most recent version at doi:10.1101/gad.291004

\section{Supplemental http://genesdev.cshlp.org/content/suppl/2004/04/05/18.8.901.DC1 Material}

References This article cites 48 articles, 13 of which can be accessed free at: http://genesdev.cshlp.org/content/18/8/901.full.html\#ref-list-1

\section{License}

Email Alerting

Receive free email alerts when new articles cite this article - sign up in the box at the top Service right corner of the article or click here.

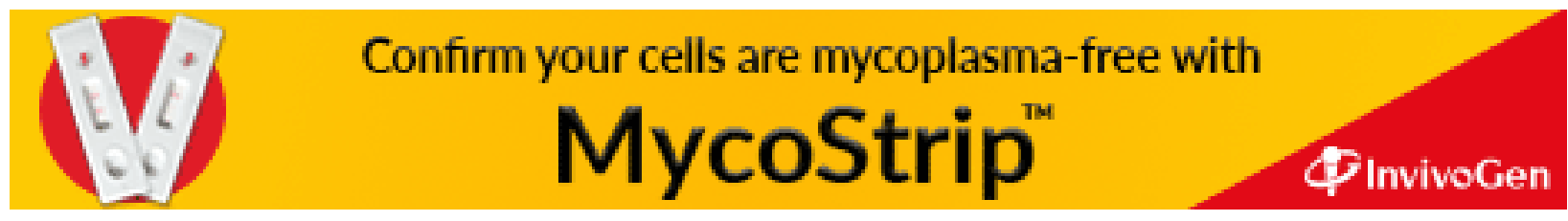

